\title{
$\eta$ meson production in nucleon-nucleon collisions within an effective Lagrangian model
}

\author{
R. Shyam \\ Saha Institute of Nuclear Physics, Kolkata 70064, India
}

(Dated: October 17, 2018)

\begin{abstract}
We investigate the $p p \rightarrow p p \eta$ and $p n \rightarrow p n \eta$ reactions within an effective Lagrangian model for laboratory kinetic energies ranging from very close to the eta meson production threshold to about $10 \mathrm{GeV}$. Production amplitudes include contributions from the mechanism of excitation, propagation and decay of $N^{*}(1535), N^{*}(1650)$, and $N^{*}(1710)$ baryonic resonances. The initial interaction between two incoming nucleons is modeled by the exchange of $\pi, \rho, \omega$ and $\sigma$ mesons where the vertex parameters are taken to be the same as those used in the previous applications of this model. Parameters of the resonance vertices also have been taken from our earlier studies wherever applicable. Calculations have been done for total as well as differential $\eta$ production cross sections. To describe the data for energies closer to the production threshold final state interactions among the out-going particles have been included by means of a generalized WatsonMigdal method. Terms corresponding to the excitation of $N^{*}(1535)$ resonance and pion exchange process dominate the cross sections. With a single set of vertex parameters our model is able to describe the available data well on total cross sections for beam energies ranging from close to threshold to upto $10 \mathrm{GeV}$.

PACS numbers: 13.60.Le, 13.75.Cs, 11.80. - m, 12.40.Vv
\end{abstract}




\section{INTRODUCTION}

The low energy behavior of the quantum chromodynamics (QCD) is not accessible to the perturbative approaches; the lattice gauge theory [1] is the ideal tool for this purpose. Despite enormous computational power necessary for the numerical realization, lattice QCD calculations have started, very recently, to describe masses and other constants of the baryonic ground as well as excited states [2]. Experimentally, the determination of baryonic resonance properties proceeds indirectly by exciting the nucleon with the help of a hadronic or electromagnetic probe and performing measurements for their decay products (mesons and nucleons). The reliable extraction of nucleon resonance properties from such experiments is a major issue of the hadron physics.

In recent years, important advances have been made in the experimental investigation of meson production reactions in nucleon-nucleon $(N N)$ collisions, particularly at beam energies very close to respective production thresholds [3, 4, 5, 6, 7, 8, 9, 10, 11, 12, 13, 14, 15]. Low incident energies also provide the opportunity to investigate the meson-nucleon interactions through these reactions since in this energy regime the final state interactions among the outgoing particles affect strongly the meson production cross sections.

The $\eta$ meson, which is the next lightest nonstrange member in the meson mass spectrum, has been a subject of considerable interest. It has been thought of as a probe for the $s \bar{s}$ component in the nucleon wave function [16]. There is also interest in measuring the rare decays of $\eta$ which could provide a new rigorous test of the standard model [17] or even of the physics beyond this. The nucleon resonance $N^{*}(1535)\left[S_{11}(1535)\right]$ with spin $\frac{1}{2}$, isospin $\frac{1}{2}$, and odd parity, has a remarkably large $\eta N$ branching ratio. It lies very close to the threshold of the $N N \rightarrow N N \eta$ reaction and contributes to the amplitude of this reaction even at the threshold. Therefore, the study of $\eta$-meson production in $N N$ collisions at the near threshold beam energies provides the unique opportunity to investigate the properties of $N^{*}(1535)$ which have been the subject of some debate recently (see, e.g., [18]). The attractive nature of the $\eta$-nucleon interaction may lead to the formation of bound (quasibound) $\eta$-nucleus states (see, e.g., [19, 20, 21, 22, 23]). This subject has been a topic of intense discussion at a recent work shop [24].

Production of $\eta$ meson in heavy ion collisions also is of great interest. Due to the high threshold of the elementary production reaction, $\eta$ mesons in such collisions are produced 
only by very energetic nucleons and reflect therefore, the tails of the nucleon momentum distributions as they arise in a high density and high temperature phase of the collision [25]. The elementary $N N \eta$ production cross sections are a crucial ingredient in the transport model studies of the $\eta$-meson production in the nucleus-nucleus collisions.

Since lattice QCD calculations are still far from being amenable to the low and intermediate energy scattering and reaction processes, the effective methods are mostly used for the description of the dynamics of the meson production reactions in hadronic collisions. These approaches introduce the baryonic resonance states explicitly in their framework and try to extract their properties by comparing the theoretical results with experimental observables. Several authors have used models of such type in describing the $\eta$ meson production in $N N$ collisions [26, 27, 28, 29, 30, 31, 32].

The main objective of this paper is to investigate the $\eta$ meson production in $N N$ collisions in the framework of an effective Lagrangian Model (ELM) which has been used earlier rather successfully to describe the pion [33, 34], associate kaon [35], and dilepton [36] production data in such collisions. The motivation here is to see as to how far can one explain the recently measured data on total and differential cross sections of $p p \rightarrow p p \eta$ [11, 12, 13, 14, 15] and the $p n \rightarrow p n \eta[10]$ reactions within this model using same sets of entrance channel and resonance channel parameters (for those resonances that appeared in earlier applications [35, 36]).

Within the ELM, initial interaction between two incoming nucleons is modeled by an effective Lagrangian which is based on the exchange of $\pi, \rho, \omega$ and $\sigma$ mesons. The coupling constants at the nucleon-nucleon-meson vertices are determined by directly fitting the $T$ matrices of the $N N$ scattering in the relevant energy region [37]. The effective Lagrangian uses the pseudovector (PV) coupling for the nucleon-nucleon-pion vertex, as it is consistent with the chiral symmetry requirement of the QCD [38, 39, 40] and also it leads to negligible contributions from the negative energy states ("pair suppression phenomena") [41]. The $\eta$ meson production proceeds via excitation of $N^{*}(1535), N^{*}(1650)$ and $N^{*}(1710)$ intermediate baryonic resonance states which have known branching ratios for the decay into the $\eta N$ channel. The coupling constants at the resonance-nucleon meson vertices are determined from the experimental widths for the decay of the resonances into the relevant channels except for those involving the $\omega$ meson where they are determined from the vector meson dominance (VMD) hypothesis. The interference terms between amplitudes corresponding 
to various meson exchanges and the intermediate resonance states have been included.

The final state interaction (FSI) among the outgoing particles affect strongly the cross sections of the $N N \rightarrow N N \eta$ reaction at near threshold beam energies [29, 30, 32]. In applications of the ELM to describe the near threshold meson production reactions in $N N$ collisions [34, 35], the FSI effects were included within the Watson-Midgal theory [42] which is based on the assumption that the FSI effects are strong in relation to the production process and that they occur attractively between only one particular pair of out-going particles. In Ref. [35], this method was used somehow arbitrarily for all the 3 outgoing pairs of particles. However, Watson's method as such is not applicable, in strict sense, for those cases where the attraction between outgoing particles is not so pronounced or where interaction between more than one pair is to be included in calculations. In this paper, we employ a generalized Watson method in which three-body states are treated by splitting the total interaction into pairwise net interactions which leads to a series decomposition of the net scattering among all the particles in terms of separate total scattering between pairs of particles (see, e.g. [43]). However, the three-body interactions are neglected. In view of the arguments presented in Refs. [32, 44] in favor of using the three-body scattering theory to describe the $N N \eta$ process, it would be interesting to see to what extent this generalized method is able to explain the $N N \eta$ production data.

In the next section, we present a brief description of the ELM where we describe the main ingredients of the theory and give all the input parameters used in our calculations. The generalized Watson method of FSI effects is also described in a subsection here. The results of calculations and their discussions are presented in section III. Summary and conclusions of our work are given in section IV.

\section{FORMALISM}

A representative of the lowest order Feynman diagrams contributing to the $\eta$ meson production considered by us, is shown in Fig. 1. Momenta of various particles are indicated in Fig. 1a. $q, p_{i}$, and $p_{\eta}$ are four momenta of the exchanged meson, the intermediate resonance and the $\eta$-meson, respectively. To evaluate various amplitudes, we have used the effective Lagrangians for the nucleon-nucleon-meson and resonance-nucleon-meson vertices as described below. 


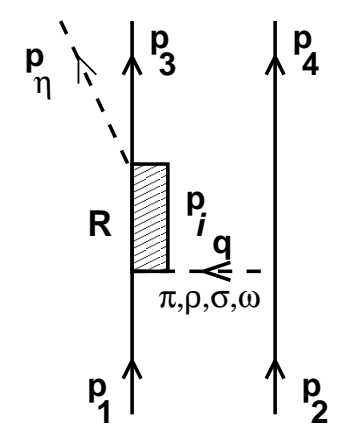

(a)

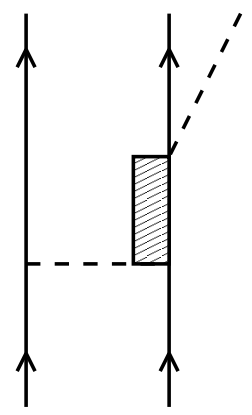

(b)

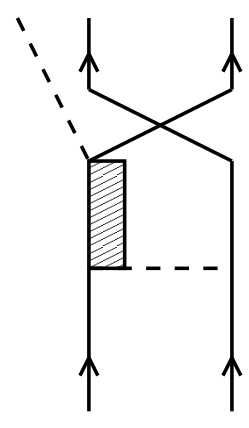

(c)

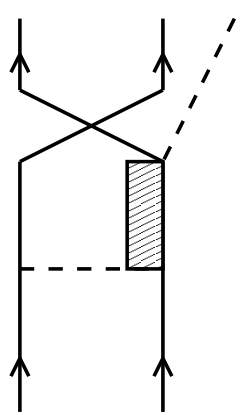

(d)

FIG. 1: Feynman diagrams for the $\eta$-meson production in nucleon-nucleon collisions. Diagrams (a) and (b) show the direct processes, while (c) and (d) the exchange ones. $R$ represents a baryonic resonance.

\section{A. Nucleon-nucleon-meson vertex}

As done before in the investigation of $p p \rightarrow p p \pi^{0}, p p \rightarrow p n \pi^{+}[33], p p \rightarrow p \Lambda K^{+}[35]$, and $N N \rightarrow N N e^{+} e^{-}[36]$ reactions, the parameters for nucleon-nucleon-meson vertices are determined by fitting the $N N$ elastic scattering $T$ matrix with an effective $N N$ interaction based on the $\pi, \rho, \omega$, and $\sigma$ meson exchanges. The effective $N N m e s o n$ Lagrangians are (see, e.g., [45, 46])

$$
\begin{aligned}
\mathcal{L}_{N N \pi} & =-\frac{g_{N N \pi}}{2 m_{N}} \bar{\Psi}_{N} \gamma_{5} \gamma_{\mu} \boldsymbol{\tau} \cdot\left(\partial^{\mu} \boldsymbol{\Phi}_{\pi}\right) \Psi_{N} . \\
\mathcal{L}_{N N \rho} & =-g_{N N \rho} \bar{\Psi}_{N}\left(\gamma_{\mu}+\frac{k_{\rho}}{2 m_{N}} \sigma_{\mu \nu} \partial^{\nu}\right) \boldsymbol{\tau} \cdot \boldsymbol{\rho}^{\mu} \Psi_{N} . \\
\mathcal{L}_{N N \omega} & =-g_{N N \omega} \bar{\Psi}_{N}\left(\gamma_{\mu}+\frac{k_{\omega}}{2 m_{N}} \sigma_{\mu \nu} \partial^{\nu}\right) \omega^{\mu} \Psi_{N} . \\
\mathcal{L}_{N N \sigma} & =g_{N N \sigma} \bar{\Psi}_{N} \sigma \Psi_{N} .
\end{aligned}
$$

In Eqs. (1)-(4), we have used the notations and conventions of Bjorken and Drell [47] and definitions of various terms are the same as those given there. In Eq. (1) $m_{N}$ denotes the 
nucleon mass. It should be noted that we use a PV coupling for the $N N \pi$ vertex. Since these Lagrangians are used to directly model the $N N T$ matrix, we have also included a nucleon-nucleon-axial-vector-isovector vertex, with the effective Lagrangian given by

$$
\mathcal{L}_{N N A}=g_{N N A} \bar{\Psi} \gamma_{5} \gamma_{\mu} \tau \Psi \cdot \mathbf{A}^{\mu}
$$

where A represents the axial-vector meson field. This term is introduced because if the mass of the axial meson $A$ is chosen to be very large $\left(\gg m_{N}\right)$ [37] and $g_{N N A}$ is defined as

$$
g_{N N A}=\frac{1}{\sqrt{3}} m_{A}\left(\frac{g_{N N \pi}}{2 m_{N}}\right)
$$

it cures the unphysical behavior in the angular distribution of $N N$ scattering caused by the contact term in the one-pion exchange amplitude. It should be mentioned here that $A$ is different from the $a_{1}(1260)$ meson resonance. The role of the $A$ vertex is to explicitly subtract out the contact term of the one-pion exchange part of the $N N$ interaction. Similar term in the coordinate space potential is effectively switched off by the repulsive hard core.

At each interaction vertex, the following form factor is introduced

$$
F_{i}^{N N}=\left(\frac{\lambda_{i}^{2}-m_{i}^{2}}{\lambda_{i}^{2}-q_{i}^{2}}\right), i=\pi, \rho, \sigma, \omega,
$$

where $q_{i}$ and $m_{i}$ are the four momentum and mass of the $i$ th exchanged meson and $\lambda_{i}$ is the corresponding cut-off parameter. The latter governs the range of suppression of the contributions of high momenta which is done via the form factor. Since $N N$ scattering cross sections decrease gradually with the beam energy (beyond certain value), and since we fit the elastic $T$ matrix directly, the coupling constants are expected to be energy dependent. Therefore, while keeping the cut-offs $\lambda_{i}$ [in Eq. (7)] energy independent, we take energy dependent meson-nucleon coupling constants of the following form

$$
g(\sqrt{s})=g_{0} \exp (-\ell \sqrt{s})
$$

where $s$ is the square of the total CM energy. The parameters $g_{0}, \ell$, and $\lambda$ were determined by fitting to the $T$ matrix of the relevant proton-proton and proton-neutron scattering data at the beam energies in the range of $800 \mathrm{MeV}$ to $4.00 \mathrm{GeV}$ [37]. This procedure also fixes signs of the effective Lagrangians [Eqs. (1)-(5)]. The values of various parameters are shown in Table I [the signs of all the coupling constants (g) are positive]. In this table the parameters of the $A$ exchange vertex are not explicitly shown as they are related to those of the pion 
via Eq. (6). We would like to remark that the same parameters were also used to describe the initial $N N$ interaction in the calculations reported in Refs. [33, 35, 36]. This ensures that the elastic $N N$ elastic scattering channel remains the same in the description of various

TABLE I: Coupling constants for the $N N$-meson vertices used in the calculations

\begin{tabular}{|c|c|c|c|c|}
\hline Meson & $g^{2} / 4 \pi$ & $\ell$ & $\begin{array}{c}\Lambda \\
(\mathrm{GeV})\end{array}$ & $\begin{array}{l}\text { mass } \\
(\mathrm{GeV})\end{array}$ \\
\hline$\pi$ & 12.562 & 0.1133 & 1.005 & 0.138 \\
\hline$\sigma$ & 2.340 & 0.1070 & 1.952 & 0.550 \\
\hline$\omega$ & 46.035 & 0.0985 & 0.984 & 0.783 \\
\hline$\rho$ & 0.317 & 0.1800 & 1.607 & 0.770 \\
\hline$k_{\rho}=6.033, k_{\omega}=0.0$ & & & & \\
\hline
\end{tabular}

inelastic processes within this model.

The main criterion for choosing the meson exchanges as discussed above is to describe the $N N$ scattering in the relevant beam energy region. We have left out the $\eta$-meson exchange in our description of the $N N$ interaction since due to the psuedoscalar (PS) nature of its coupling, the contribution of the exchange terms of this particle (having a mass much larger than that of the psuedoscalar pion) is expected to be very small. Furthermore, as confirmed by several studies (see, e.g., [48, 49, 50, 51]), the coupling constant for the $N N \eta$ vertex is rather small.

\section{B. Resonance-nucleon-meson vertex}

As the $\eta$ meson has zero isospin, only isospin- $\frac{1}{2}$ nucleon resonances are allowed. Below $2 \mathrm{GeV}$ center of mass (c.m.) energy, $N^{*}(1535)$ has a prominent decay branching ratio of 40-60\% into the $N \eta$ channel (see, e.g., the latest review of the particle data group [52]). On the other hand, $N^{*}(1650)$ and $N^{*}(1710)$ resonances have a small but non-negligible decay branching ratios of $3-10 \%$ and $6 \pm 1 \%$, respectively, to this channel. In comparison to these, the branching ratio for the decay of $N^{*}(1520)$ resonance to $N \eta$ channel is negligibly small and we have not included it in out description. In several previous studies of the $N N \rightarrow N N \eta$ reaction, contributions from only $N^{*}(1535)$ resonance have been considered. 
Since all the three resonances can couple to the meson-nucleon channels considered in the previous section, we require the effective Lagrangians for all the four resonance-nucleonmeson vertices corresponding to all the included resonances. At the spin- $\frac{1}{2}$ resonances $N-\pi(\eta)$ vertices, we have the choice of PS or PV couplings. The corresponding effective Lagrangians can be written as [35, 48, 53, 54]

$$
\begin{aligned}
& \mathcal{L}_{N_{1 / 2}^{*} N \pi}^{P V}=-\frac{g_{N_{1 / 2}^{*} N \pi}}{M} \bar{\Psi}_{N^{*}} \Gamma_{\mu} \boldsymbol{\tau} \cdot\left(\partial^{\mu} \boldsymbol{\Phi}_{\pi}\right) \Psi_{N}+\text { h.c. } \\
& \mathcal{L}_{N_{1 / 2}^{*} N \pi}^{P S}=-g_{N_{1 / 2}^{*} N \pi} \bar{\Psi}_{N^{*}} i \Gamma \boldsymbol{\tau} \boldsymbol{\Phi}_{\pi} \Psi_{N}+\text { h.c. } \\
& \mathcal{L}_{N_{1 / 2}^{*} N \eta}^{P V}=-\frac{g_{N_{1 / 2}^{*} \eta}}{M} \bar{\Psi}_{N^{*}} \Gamma_{\mu} \boldsymbol{\tau} \cdot\left(\partial^{\mu} \boldsymbol{\Phi}_{\eta}\right) \Psi_{N}+\text { h.c. } \\
& \mathcal{L}_{N_{1 / 2}^{*} N \eta}^{P S}=-g_{N_{1 / 2}^{*} N \eta} \bar{\Psi}_{N^{*}} i \Gamma \boldsymbol{\tau} \boldsymbol{\Phi}_{\eta} \Psi_{N}+\text { h.c. }
\end{aligned}
$$

where $M=\left(m_{N^{*}} \pm m_{N}\right)$, with upper sign for even parity and lower sign for odd parity resonance. The operators $\Gamma, \Gamma_{\mu}$, are given by,

$$
\begin{array}{r}
\Gamma=\gamma_{5}, \Gamma_{\mu}=\gamma_{5} \gamma_{\mu}, \\
\Gamma=1, \Gamma_{\mu}=\gamma_{\mu},
\end{array}
$$

for resonances of even and odd parities, respectively. We have performed calculations with both of these couplings. The effective Lagrangians for the coupling of resonances to other mesons are,

$$
\begin{aligned}
\mathcal{L}_{N_{1 / 2}^{*} N \rho} & =-g_{N_{1 / 2}^{*} N \rho} \bar{\Psi}_{N^{*}} \frac{1}{2 m_{N}} \Gamma_{\mu \nu} \partial^{\nu} \boldsymbol{\tau} \cdot \boldsymbol{\rho}^{\mu} \Psi_{N} \cdot+\text { h.c. } \\
\mathcal{L}_{N_{1 / 2}^{*} N \omega} & =-g_{N_{1 / 2}^{*} N \omega} \bar{\Psi}_{N^{*}} \frac{1}{2 m_{N}} \Gamma_{\mu \nu} \partial^{\nu} \omega^{\mu} \Psi_{N} .+ \text { h.c. } \\
\mathcal{L}_{N_{1 / 2}^{*} N \sigma} & =g_{N_{1 / 2}^{*} N \sigma} \bar{\Psi}_{N^{*}} \Gamma^{\prime} \sigma \Psi_{N}+\text { h.c. }
\end{aligned}
$$

where operators $\Gamma^{\prime}$ and $\Gamma_{\mu \nu}$ are,

$$
\begin{gathered}
\Gamma^{\prime}=1, \Gamma_{\mu \nu}=\sigma_{\mu \nu} \\
\Gamma^{\prime}=\gamma_{5}, \Gamma_{\mu \nu}=\gamma_{5} \sigma_{\mu \nu},
\end{gathered}
$$

for resonances of even and odd parities, respectively.

We assume that the off-shell dependence of the $N N^{*}$ vertices are determined solely by multiplying the vertex constants by form factors. Similar to Refs. [46, 55], we use the following form factors for $N^{*} N$-meson vertices

$$
F_{i}^{N N^{*}}=\left[\frac{\left(\lambda_{i}^{N^{*}}\right)^{4}}{\left(\lambda_{i}^{N^{*}}\right)^{4}+\left(q_{i}^{2}-m_{i}^{2}\right)^{2}}\right], i=\pi, \rho, \sigma, \omega,
$$


The resonance couplings are determined from the experimentally observed branching ratios for the decay of the resonances to the corresponding channels. Since the resonances considered in this study have no known branching ratios for the decay into the $N \omega$ channel, we determine the coupling constants for the $N^{*} N \omega$ vertices by the strict vector meson dominance (VMD) hypothesis [56], which is based essentially on the assumption that the coupling of photons to hadrons takes place through a vector meson. For details of these calculations we refer to [35].

The resonance properties and the values of various coupling constants are given in Table II. Value of the cut-off parameter $\left(\lambda_{i}^{N^{*}}\right)$ is taken to be $1.2 \mathrm{GeV}$ for all the vertices, which is the same as that used in Refs. [36, 55]. Fixing $\lambda_{i}^{N^{*}}$ to one value minimizes the number of free parameters.

It should however, be stresses that the branching ratios determine only the square of the corresponding coupling constants, thus their signs remain uncertain in this method. Predictions from independent studies are used as a guide to fix these signs. We have followed here the results of Ref. [46] for this purpose. The propagators for various mesons and nucleon resonances in the calculation of the amplitudes have been taken to be the same as those discussed in [35, 36].

\section{Amplitudes and cross sections}

After having established the effective Lagrangians, coupling constants and form of the propagators, the amplitudes for various diagrams associated with the $N N \rightarrow N N \eta$ reaction can be calculated in straight forward manner by following the well known Feynman rules. The isospin part is treated separately. This gives rise to a constant factor for each graph, which is shown in Table III.

It should be stressed here that signs of various amplitudes are fixed, by those of the effective Lagrangians, coupling constants and propagators as described above. These signs are not allowed to change anywhere in the calculations.

The general formula for the invariant cross section of the $N N \rightarrow N N \eta$ reaction is written as

$$
d \sigma=\frac{m_{N}^{4}}{2 \sqrt{\left[\left(p_{1} \cdot p_{2}\right)^{2}-m_{N}^{4}\right]}} \frac{1}{(2 \pi)^{5}} \delta^{4}\left(P_{f}-P_{i}\right)\left|T_{f i}\right|^{2} \prod_{a=1}^{3} \frac{d^{3} p_{a}}{E_{a}},
$$


TABLE II: Resonance parameters and the coupling constants for various decay vertices. Coupling constants at the $N^{*} N \omega$ vertices are obtained from the vector meson dominance hypothesis (see, e.g. Ref. [35]).

\begin{tabular}{|c|c|c|c|}
\hline Resonance & $\begin{array}{l}\text { Width } \\
(\mathrm{GeV})\end{array}$ & Decay channel & $g$ \\
\hline \multirow[t]{5}{*}{$N^{*}(1535)$} & 0.150 & $N \pi$ & 0.6840 \\
\hline & & $N \rho$ & 3.9497 \\
\hline & & $N \omega$ & 1.4542 \\
\hline & & $N \sigma$ & 2.5032 \\
\hline & & $N \eta$ & 2.2000 \\
\hline \multirow[t]{5}{*}{$N^{*}(1650)$} & 0.150 & $N \pi$ & 0.8096 \\
\hline & & $N \rho$ & 2.6163 \\
\hline & & $N \omega$ & 1.8013 \\
\hline & & $N \sigma$ & 2.5032 \\
\hline & & $N \eta$ & -0.5469 \\
\hline \multirow[t]{5}{*}{$N^{*}(1710)$} & 0.150 & $N \pi$ & 1.0414 \\
\hline & & $N \rho$ & 2.9343 \\
\hline & & $N \omega$ & 1.5613 \\
\hline & & $N \sigma$ & 0.6737 \\
\hline & & $N \eta$ & 1.0328 \\
\hline
\end{tabular}

where $T_{f i}$ represents the total amplitude, $P_{i}$ and $P_{f}$ the sum of all the momenta in the initial and final states, respectively, and $p_{a}$ the momenta of the three particles in the final state. The corresponding cross sections in the laboratory or center of mass systems can be written from this equation by imposing the relevant conditions.

\section{Final state interaction}

For describing the data for the $N N \rightarrow N N \eta$ reaction at beam energies very close to the $\eta$ production threshold, consideration of the final state interaction (FSI) among the 
TABLE III: Isospin factors for various diagrams, Isovector corresponds to $\pi$ and $\rho$ exchange graphs while isoscalar to $\omega$ and $\sigma$ ones

\begin{tabular}{|ccc|}
\hline \hline graph & isovector & isoscalar \\
\hline \multirow{2}{*}{ direct } & $p p \rightarrow p p \eta$ & \\
exchange & 1.0 & 1.0 \\
& 1.0 & 1.0 \\
direct & $p n \rightarrow p n \eta$ & 1 \\
exchange & -1 & 0 \\
\hline \hline
\end{tabular}

three out going particles is important. We follow here an approximate scheme in line with the Watson-Migdal theory of FSI [42]. In this approach the energy dependence of the cross section due to FSI is separated from that of the primary production amplitude. This is based on the assumption that the reaction takes place over a small region of space, a condition fulfilled rather well in near threshold reactions involving heavy mesons. This method has been extensively applied to study the low momentum behavior of the pion [34, 57, 58], $\eta$ meson [59, 60, 61] , associated hyperon [35, 62] and $\phi$-meson [63] production in $N N$ collisions. The total amplitude is written as

$$
T_{f i}=T_{0}(N N \rightarrow N N \eta) \cdot T_{f f}
$$

where $T_{0}(N N \rightarrow N N \eta)$ is the primary production amplitude, while $T_{f f}$ describes the rescattering among the final particles which goes to unity in the limit of no FSI. The factorization of the total amplitude into those of the FSI and primary production (Eq. (22)), enables one to pursue the diagrammatic approach for the latter within an effective Lagrangian model and investigate the role of various meson exchanges and resonances in describing the reaction.

Watson's original method [42] was developed for those cases where the final state interaction is strong in relation to the production process and where it is confined only to one particular pair of particles (mostly among nucleons in case of nucleon-nucleon-meson final states). On the other hand, in certain cases it may be necessary to include FSI among all the three outgoing particles since even if the meson-baryon interactions are weak, they can 
still be influential through interference. In Ref. [35], the $T$ matrix $T_{f f}$ was written (without providing any proof) as a coherent sum of the transition matrices describing the final state interaction among all three two-body subsystems of the final state nucleon-nucleon-meson system. We show here that this result can be obtained (in a slightly different form) by following the technique of multiple FSI as discussed in Ref. [43]. To that end, we first try to get a representation for the total amplitude $T_{f i}$ in terms of an expression similar to that given by Eq. (22) where the FSI amplitude $T_{f f}$ is appropriately constructed.

To introduce the treatment of the FSI for the three-particle system, we assume that the three-particle final states can be represented by additive potentials of the form $U=$ $U_{12}+U_{31}+U_{23} \equiv U_{3}+U_{2}+U_{1}$. With this assumption, the total amplitude $T_{f i}$ can be written as a iteration series in terms of the production amplitude $T_{0}$ (defined in Eq. (22)) and the three-body final state pair interaction amplitudes $T_{i j} \equiv T_{k}$ (see Appendix A for details).

$$
T_{f i}=T_{0}+\sum_{k} T_{k} G_{0} T_{0}+\sum_{k \neq j} T_{k} G_{0} T_{j} G_{0} T_{0}+\ldots,
$$

where the final state interaction transition matrices $T_{k}$ are as defined in Appendix A. $G_{0}$ is the Green's function corresponding to the free Hamiltonian (kinetic energy). Neglecting processes depicted by the third term, this decomposition can be expressed by Fig. 2 . It is easy to show that the result of the Watson FSI theory are recovered if one retains only the amplitude $T_{0}$ and a single pair amplitude, say, $T_{12}$.

Any practical calculation requires evaluation of the matrix element < 123|T $T_{12} G_{0} T_{0} \mid N_{1} N_{2}>$, where $N_{1}$ and $N_{2}$ denote two particles of the incident channel and 1,2 and 3 represent the outgoing channel particles. Introducing a complete set of intermediate states of particles, say, 1 and 2, we get for this channel

$$
T_{f i}=\frac{1}{(2 \pi)^{3}} \int \frac{d^{3} \mathbf{k}_{1}^{\prime}}{2 E_{1}^{\prime}} \frac{d^{3} \mathbf{k}_{2}^{\prime}}{2 E_{2}^{\prime}} \delta^{3}\left(\mathbf{k}_{1}^{\prime}+\mathbf{k}_{2}^{\prime}-\mathbf{k}_{1}-\mathbf{k}_{2}\right) \frac{<\mathbf{k}_{1} \mathbf{k}_{2}\left|T_{12}\right| \mathbf{k}_{1}^{\prime} \mathbf{k}_{2}^{\prime}><\mathbf{k}_{1}^{\prime} \mathbf{k}_{2}^{\prime} \mathbf{k}_{\mathbf{3}}\left|T_{0}\right| N_{1} N_{2}>}{E-\left(E_{3}+E_{1}^{\prime}+E_{2}^{\prime}\right)+i \epsilon} .
$$

It may be noted that in this form, each particle lies on the mass shell and three momentum is conserved in the intermediate processes. Introducing the total and relative momenta, $\mathbf{p}^{\prime}=\mathbf{k}_{1}^{\prime}+\mathbf{k}_{2}^{\prime}, 2 q^{\prime}=\mathbf{k}_{1}^{\prime}-\mathbf{k}_{2}^{\prime}$, and evaluating the integral in the barycentric frame of 1 and 2 , we obtain,

$$
T_{f i}=\frac{1}{(2 \pi)^{3}} \int \frac{T_{12}\left(\xi, \theta ; \xi^{\prime}, \theta^{\prime}\right) T_{0}\left(\xi^{\prime}, \theta^{\prime}, \mathbf{k}_{3} ; \mathbf{k}_{i}\right)}{\xi-\xi^{\prime}+i \epsilon} \frac{2 q^{\prime} d \xi^{\prime} d \Omega^{\prime}}{\xi^{\prime}}
$$




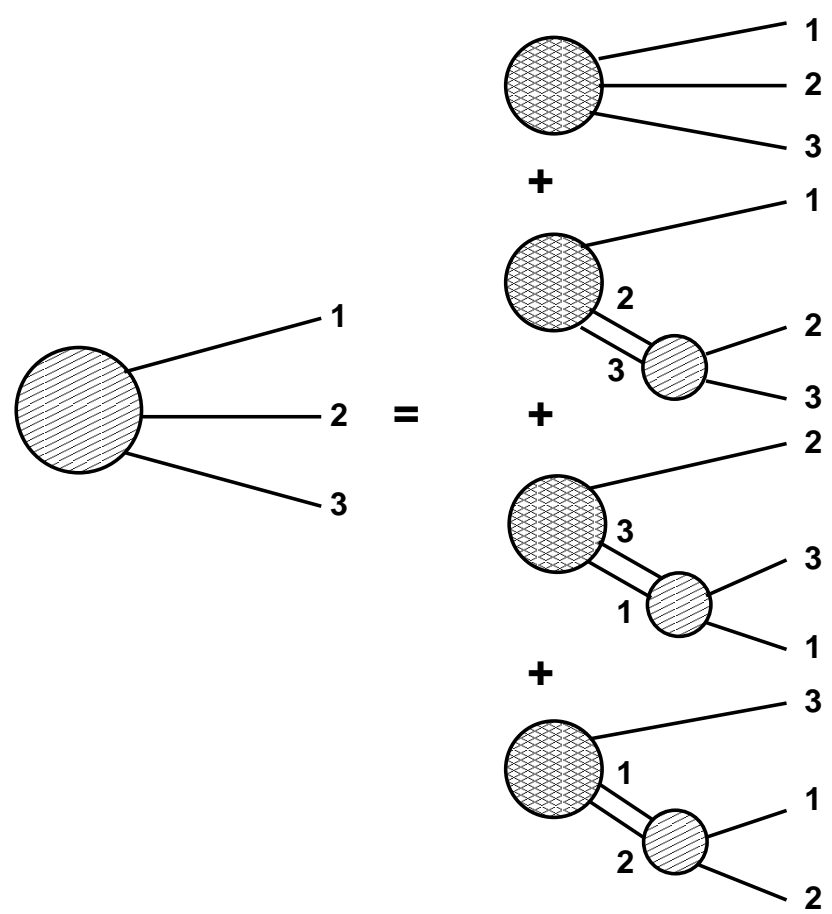

FIG. 2: Final state scattering among three particles evaluated to the lowest order

where $\xi$ is the energy of 1 and 2 in this frame, $\xi^{\prime}$ is the intermediate state and $\theta$ denotes the orientation $(\theta, \phi)$ of $\mathbf{q}$ with respect to a fixed axis.

For further evaluation of the integral, we make a partial wave decomposition of the amplitude and for each partial wave we rewrite it as

$$
T_{f i}^{\ell m} \propto T_{12}(\xi, \xi) T_{0}(\xi) \int \frac{\left[T_{12}\left(\xi, \xi^{\prime}\right) T_{0}\left(\xi^{\prime}\right) / T_{12}(\xi, \xi) T_{0}(\xi)\right]}{\xi-\xi^{\prime}+i \epsilon} \frac{2 q^{\prime} d \xi^{\prime} d \Omega^{\prime}}{\xi^{\prime}}
$$

Now we make the assumption that the ratio within the square brackets in the integrand of Eq. (26) is constant upto a certain energy $\xi_{c}$ and zero thereafter. Extending this procedure to all the three interacting pairs, we get in the low energy and $s$-wave limit

$$
T_{f i} \simeq T_{0}(\xi) T_{f f}(\xi)
$$

In Eq. (27) $T_{f f}$ is defined as

$$
T_{f f}(\xi)=\sum_{i \neq j} c_{i j} T_{i j}(\xi, \xi),
$$

where

$$
c_{i j}=\frac{1}{\pi} \cosh ^{-1}\left[1+\frac{16 m_{i} m_{j}\left(\xi_{c}-m_{i}-m_{j}\right)}{\left(m_{i}+m_{j}\right)^{3}}\right] .
$$


It is obvious that Eq. (28) allows interference among the final state scattering amplitudes. We further note that apart from the factor $c_{i j}$, this equation is similar to that used in Ref. [35].

The derivation of Eq. (27) is independent of the strength of the interaction and of whether it is attractive or repulsive. The quantity $c_{i j}$ can be regarded as the amount of final state scattering that takes place in a particular channel $i j$. A plausible value of the cut off $\xi_{c}$ comes from the constraint that for the $N N$ sub-state $c_{i j}$ should come out to be unity since in the limit of FSI in only this sub-state, we should recover Watson's result. With the same value of the $\xi_{c}$, the $c_{i j}$ for the $\eta N$ sub-state comes out to be 1.07. It must however, be noted that this procedure determines the value of the $c_{i j}$ at best only for the $N N$ channel. It remains largely undetermined for the $\eta-p$ sub-state which could even be dependent on the relative energy of this channel. Since, for the time being we do not have a definite method to determine this quantity, we have taken the same value for the parameter $\xi_{c}$ for both the sub-states.

For calculating the FSI amplitude for the $\eta N$ sub-state, we note that there are no direct measurements of the elastic $\eta N$ scattering and the information about the $\eta N$ elastic scattering amplitude is obtained by describing the $\pi N \rightarrow \eta N$ and $\gamma N \rightarrow \eta N$ data within some model. Recently, there has been suggestions to determine the $\eta N$ scattering amplitude from the studies of associated photoproduction of $\phi$ and $\eta$ mesons off the proton [64]. We adopt here the results reported in Ref. [65] where $\eta N$ scattering parameters have been obtained by fitting the $\pi N \rightarrow \pi N, \pi N \rightarrow \eta N, \gamma N \rightarrow \eta N$ data in an energy range from threshold to about $100 \mathrm{MeV}$, in a $K$ matrix method. These authors write the elastic $\eta N$ scattering $T$ matrix as

$$
T^{-1}=1 / a+\frac{r_{0}}{2} q_{\eta}^{2}+s q_{\eta}^{4}-i q_{\eta}
$$

where $q_{\eta}$ is the momentum in the $\eta N$ center of mass (c.m.) system. Seven sets of values of the parameters $a, r_{0}$ and $s$ are given in Ref. [65]. We found that the best description of the data (within the realm of our overall input parameter sets given in Tables I and II), is provided by the $\eta N$ scattering amplitudes with the parameter set, $a=0.51+i 0.26$ $\mathrm{fm}, r_{0}=-2.50-i 0.310 \mathrm{fm}$, and $s=-0.20-i 0.04 \mathrm{fm}^{3}$. It should be noted that the real part of the $\eta N$ scattering length of this parameter set $\left(a_{R}\right)$ is about half of that of the "preferred" set of Ref. [65]. A larger $a_{R}$ is also supported by the calculations presented in 


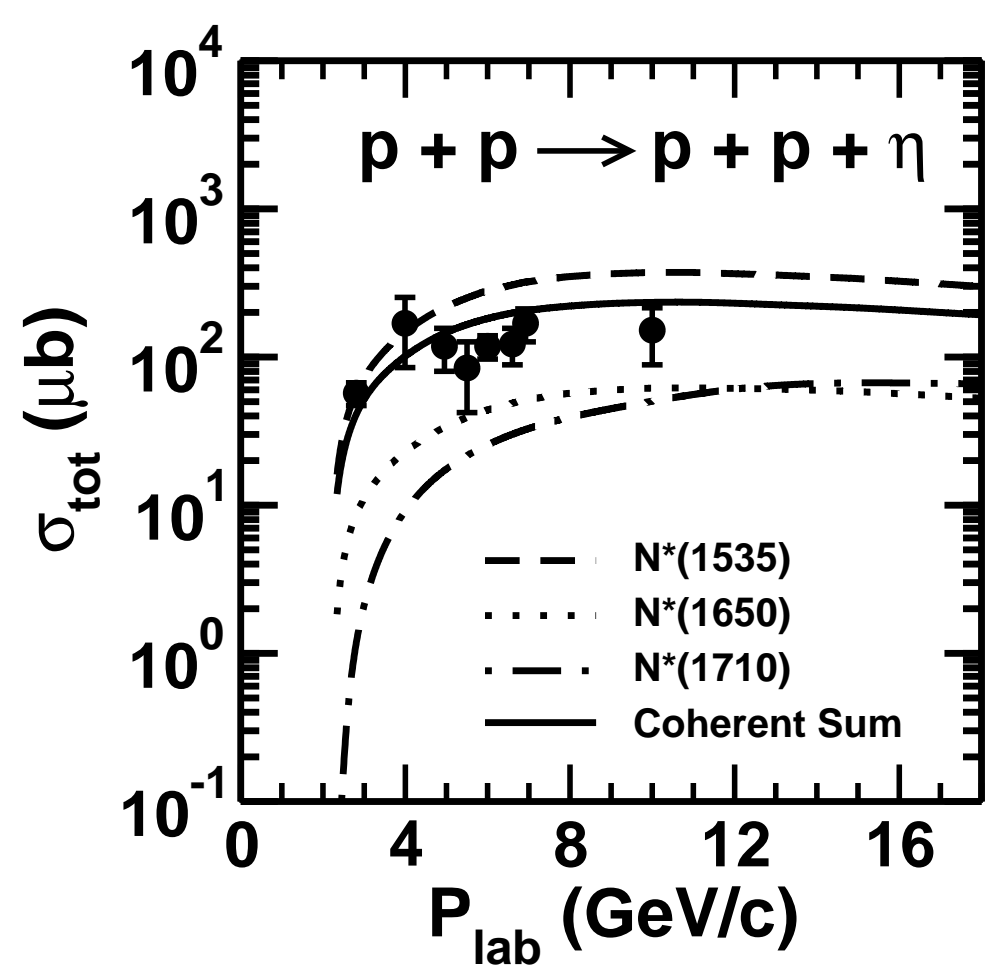

FIG. 3: The total cross section for the $p+p \rightarrow p+p+\eta$ reaction as a function of the beam momentum. The dashed, dotted and dashed-dotted curves represent the contributions of $N^{*}(1535)$, $N^{*}(1650), N^{*}(1710)$ baryonic resonance intermediate states, respectively. Their coherent sum is shown by the solid line. The experimental data are from [74].

Ref. [66]. However, we note that in the theoretical description of the $p p \rightarrow p p \eta$ reaction as reported in Refs [27, 67], the value of $a_{R}$ was similar to that used by us. A smaller $a_{R}$ is also consistent with that extracted in Ref. [54] in an effective Lagrangian model analysis of the meson-nucleon scattering. Furthermore, it was noted in Ref. [68] that within a three-body model, the shapes of the $n p \rightarrow \eta d$ cross sections can be explained over a wide energy range only with a $a_{R}$ around $0.42 \mathrm{fm}$. A smaller value of $a_{R}$ is also consistent with the Jülich model [69].

The FSI amplitude $T_{N N}$ has been calculated by following the Jost function method using the effective range expansion (ERE) of the $N N$ phase shifts as discussed in Refs. [34, 35, 42]. In case of the proton-proton sub-state, the Coulomb modified effective range expansion has been used [70]. The effective range parameters for the $N N$ channel have been taken to be the same as those used in Ref. [34].

It should however, be mentioned here that the use of on-shell forms to describe the FSI 
$T$ matrices $T_{\text {if }}$ has been criticized by some authors. It has been argued in Refs. [71, 72] that the absolute magnitudes of the cross sections obtained by such a procedure could be uncertain because of the off shell effects. Even the Jost function method has been shown [73] to produce inadequate results in an study where the scattering length parameters for the $\Lambda-p$ final state has been extracted from the $p p \rightarrow p \Lambda K^{+}$data. In the next section we have examined the role of the off shell effects in the $N N$ sub-state in some more details.

\section{RESULTS AND DISCUSSIONS}

The major aim of this paper is to check the suitability of our model and the vertex parameters appearing therein to describe the $\eta$ production cross sections over a wide range of beam energies. We have therefore, applied our approach to describe the total cross sections for the $p p \rightarrow p p \eta$ reaction for beam energies ranging from near threshold to upto $10 \mathrm{GeV}$, and for the $p n \rightarrow p n \eta$ reaction for beam energies from threshold to upto $1.6 \mathrm{GeV}$. These are the energy regimes in which experimental data are available for the two reactions. We have also used this method to describe one set of the exclusive data, namely the $\eta$ angular distributions for the former reaction. Calculations have been performed by using both the PS and PV couplings for $N^{*} N \pi$ and $N^{*} N \eta$ vertices. We note that the cross sections remain almost unchanged by switching from one type of coupling to another. In all the calculations shown below the coupling constants and cut off parameters for various vertices were the same as those discussed in section II.

A cleaner check of the vertex parameters used in calculating the amplitude $T_{0}(N N \rightarrow$ $N N \eta$ ), is provided by the comparison of our calculations with the data for beam momenta above $3 \mathrm{GeV} / \mathrm{c}$, since at these energies FSI effects are most likely to be unimportant. In Fig. 3, we show the comparison of our calculations and the experimental data (taken from Ref. [74]) for the total cross section of the $p p \rightarrow p p \eta$ reaction at higher beam energies. We notice that the measured cross sections are reproduced reasonably well by our calculations (solid line) in the entire range of beam momenta.

Individual contributions of various nucleon resonance intermediate states to the $p p \rightarrow p p \eta$ reaction are also shown in Fig. 3. Cross sections corresponding to $N^{*}(1535), N^{*}(1650)$ and $N^{*}$ (1710) resonances are represented by dashed, dotted and dashed-dotted lines, respectively while their coherent sum is shown by the solid line. We note that the contributions of 


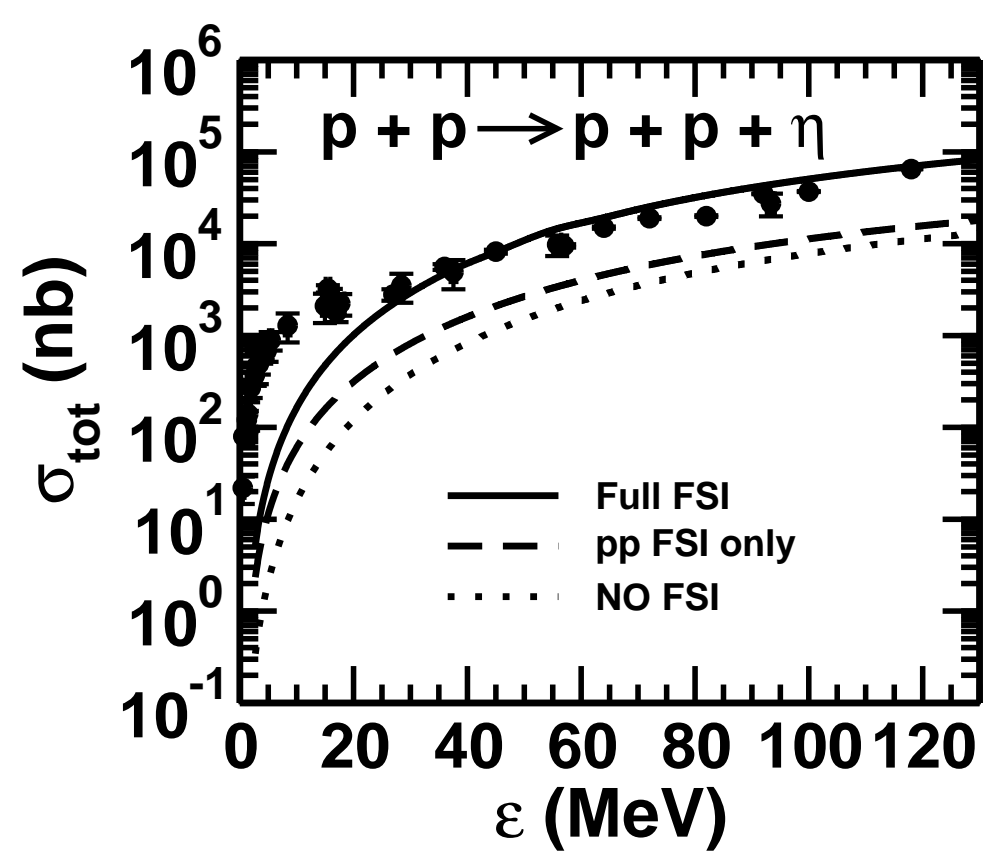

FIG. 4: The total cross section for the $p+p \rightarrow p+p+\eta$ reaction as a function of the excess energy. The dotted and dashed curves represent cross section obtained with FSI effects included only in the proton-proton sub-state of the final channel and no FSI at all, respectively. The solid line shows the results obtained with full FSI effects included as discussed in section II. The experimental data are from [11, 12, 13, 14, 15].

the $N^{*}(1535)$ resonance dominate the total cross section for all the beam momenta. In comparison, those of $N^{*}(1650)$ and $N^{*}(1710)$ resonances are smaller by factors ranging from 5 - 10. However, the interference terms of the amplitudes corresponding to various resonances are not negligible. It must again be emphasized that we have no freedom of choosing the relative signs of the interference terms.

The results shown in Fig. 3 fix the parameters of all the vertices. In the application of our model to describe $N N \eta$ data at near threshold beam energies, the amplitude $T_{0}(N N \rightarrow$ $N N \eta$ ) has been calculated with exactly the same values for all the parameters. For these energies the FSI effects in the outgoing channels, have been included by using Eqs. (27-30). The experimental cross sections in this energy regime are given as a function of the excess energy $(\epsilon)$ which is defined as $\epsilon=\sqrt{s}-2 m_{p}-m_{\eta}$, where $\sqrt{s}$ is the invariant mass.

In Figs. 4 and 5, we present comparisons of our calculations with the experimental data for total cross sections of the $p p \rightarrow p p \eta$ and $p n \rightarrow p n \eta$ reactions, respectively, as a function of $\epsilon$. 


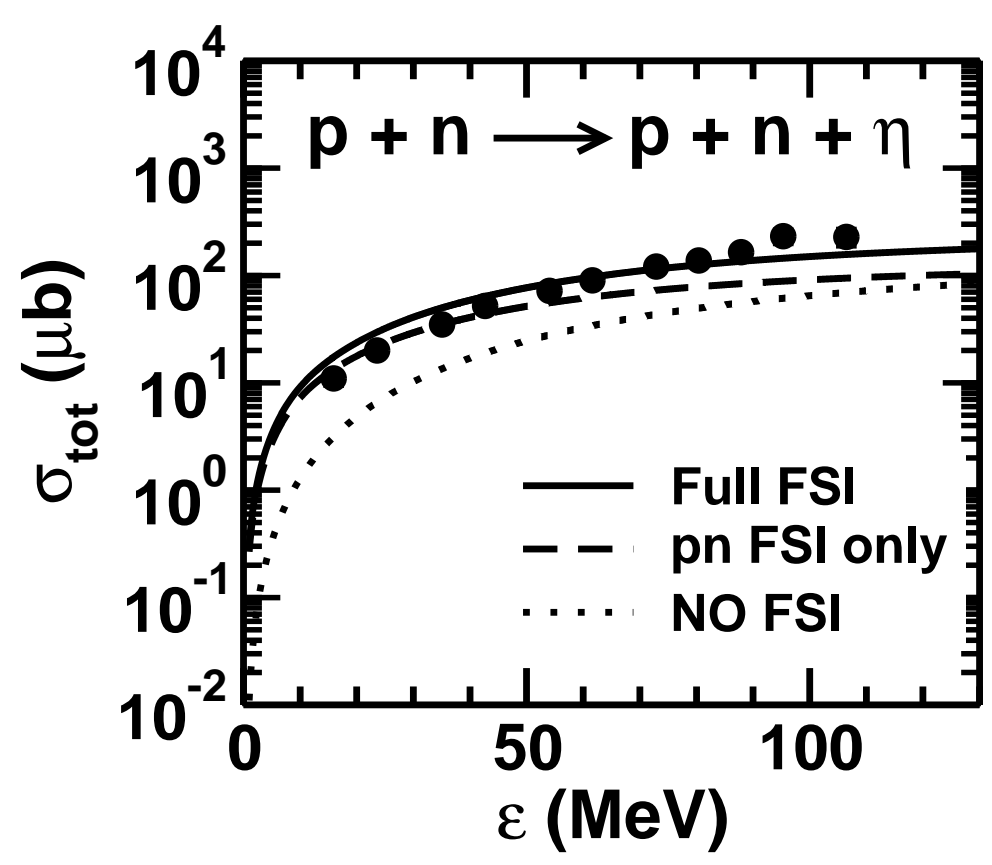

FIG. 5: The total cross section for the $p+n \rightarrow p+n+\eta$ reaction as a function of the excess energy. The dotted and dashed curves represent cross section obtained with FSI effects included only in the proton-proton sub-state of the final channel and no FSI at all, respectively. The solid line shows the results obtained with full FSI effects included as discussed in section II. The experimental data are from [10].

The solid, dashed and dotted lines show the results obtained by including the full FSI effects in all the three sub-systems, FSI only in $p p$ and $p n$ channel and no FSI at all, respectively. It should be noted that no arbitrary normalization constant has been introduced in any of the results shown in these figures. For the case of the $p p \rightarrow p p \eta$ reaction, our full calculations describe the data quite well for $\epsilon$ values in the range of 15 - $130 \mathrm{MeV}$. On the other hand, for the $p n \rightarrow p n \eta$ reaction they are in excellent agreement with the available data for all the beam energies. The FSI in the $\eta p$ sub-state is indeed quite important in our model. The difference between results obtained with FSI in only the $p p$ sub-state and that in all the three subsystems of the final channel, is comparable to that reported in the three body calculations of Ref. [32]. These authors have presented their results for $\epsilon$ values up to only $60 \mathrm{MeV}$. It would be interesting to see the results of their model also at higher values of $\epsilon$. It should, however, be noted that the description of the data for the $p n \rightarrow p n \eta$ reaction within the three-body model model is less satisfactory in comparison to that for the $p p \rightarrow p p \eta$ 


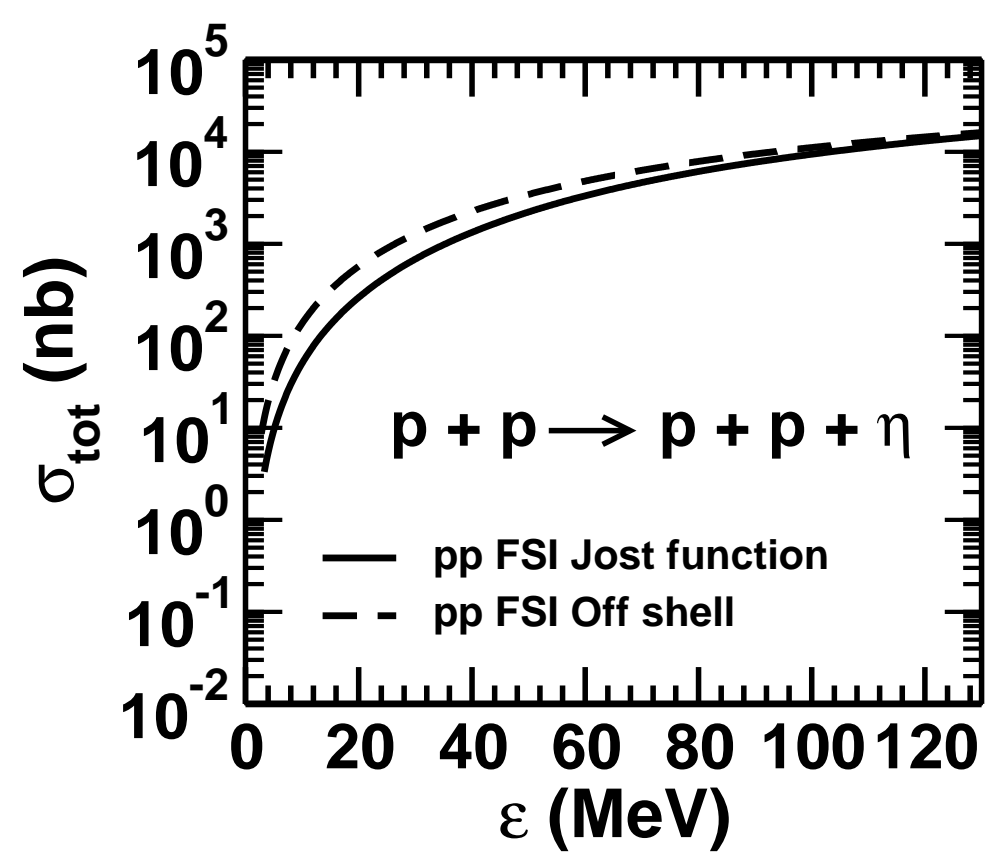

FIG. 6: The total cross section for the $p+p \rightarrow p+p+\eta$ reaction as a function of the excess energy with only the $p p$ FSI effects. The solid and dashed curves represent cross section obtained with $p p$ FSI calculated with Jost function method and that with the method described in Ref. [61].

reaction.

We see that the ELM is able to describe both the energy dependence and the absolute magnitudes of the experimental cross sections for the $\eta$ meson production in both $p p$ and $p n$ channels for excess energies $>15 \mathrm{MeV}$. However, it underpredicts the $p p$ channel data for $\epsilon$ values below $15 \mathrm{MeV}$. Such a trend has also been seen in calculations presented in Refs. [29, 30] where the $\eta$ meson production in $N N$ collisions has been investigated within a relativistic meson exchange model including the initial state interactions and FSI only in the $N N$ sub-system. These authors have attributed the near threshold underestimation of the experimental total cross section to the non-inclusion of the $\eta p$ FSI in their model.

Since the ingredients of the primary production amplitude of our model have already been checked and fixed by calculations done at higher beam energies where FSI effects are absent, the underestimation of the $p p \rightarrow p p \eta$ cross section for very low values of $\epsilon$ indicates that we need to improve the treatment of the FSI effects. Inclusion of the off shell effects in the calculations of FSI is one of the likely improvements. The knowledge about the off shell nature of the $\eta N$ interaction is still very sparse. However, we can use the results presented in 


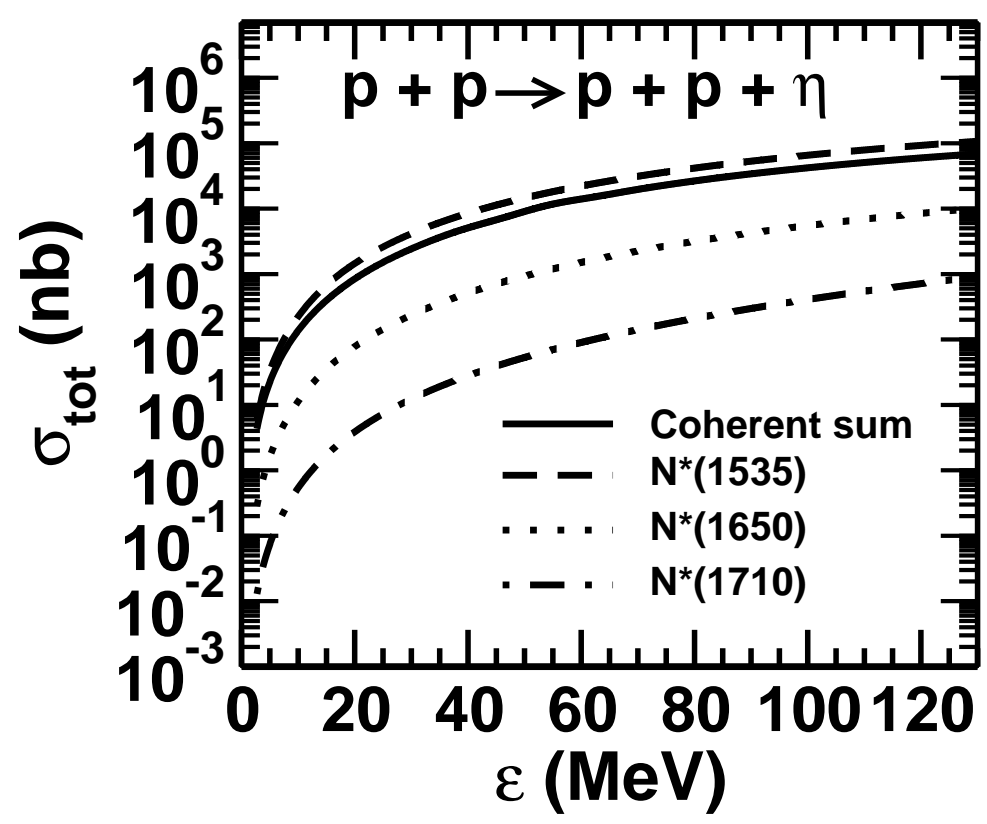

FIG. 7: Contributions of $N^{*}(1535)$ (dashed line), $N^{*}(1650)$ (dotted) and $N^{*}(1710)$ (dashed-dotted line) baryonic resonances to the total cross section for $p p \rightarrow p p \eta$ reaction. Their coherent sum is shown by the solid line.

Ref. [61] to investigate the effects of using off shell $p p$ FSI on the near threshold $\eta$ production cross sections. In Fig. 6, we have compared the results for the total cross sections for the $p p \rightarrow p p \eta$ reaction obtained by including FSI in $p p$ substate calculated within Jost function technique and that obtained with the method described in Ref. [61] which includes off-shell effects. In the results presented in this figure, no $\eta p$ FSI has been considered. We note that the off shell effects in the $p p$ FSI do increase the cross section for $\epsilon<60 \mathrm{MeV}$. However, it is not enough to explain the underprediction of the experimental data by our theory at smaller energies. One needs to have a better understanding of the $\eta N$ scattering amplitude. Further improvement may come by including the three-body terms in the expansion of the scattering amplitude given by Eq. (23). It has been shown in Ref. [32] that $N N \eta$ FSI effects calculated within a three-body scattering theory leads to enhanced cross sections at very low values of $\epsilon$.

It should be remarked here that the differences in the cross sections of $p n \rightarrow p n \eta$ and $p p \rightarrow p p \eta$ reactions are not only due to different isospin factors, but also due to differences in the FSI effects. The low energy scattering parameters between $p p$ and $p n$ cases are different; the latter involves also a triplet spin state together with the singlet one. A crucial difference 


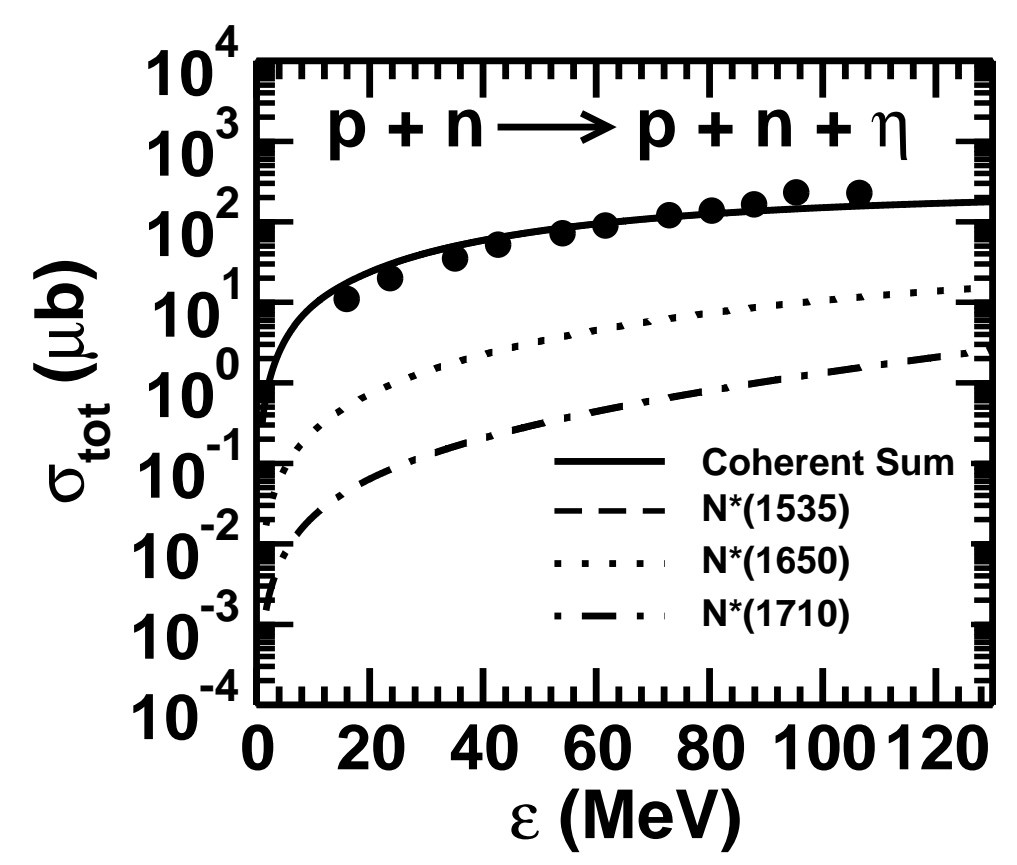

FIG. 8: Contributions of $N^{*}(1535)$ (dashed line), $N^{*}(1650)$ (dotted) and $N^{*}(1710)$ (dashed/dotted line) baryonic resonances to the total cross section for $p n \rightarrow p n \eta$ reaction. Their coherent sum is shown by the solid line.

between them is the Coulomb interaction. This is not included in the three-body model calculations of the $p p \rightarrow p p \eta$ reaction reported in Ref. [32]. Inclusion of this term is likely to reduce the cross section for beam energies very close to the threshold.

In Figs 7 and 8 we show the individual contributions of various nucleon resonances to the total cross sections of the $p p \rightarrow p p \eta$ and $p n \rightarrow p n \eta$ reactions, respectively, at the near threshold beam energies. Similar to the situation at higher beam energies, the cross sections are dominated by the $N^{*}(1535)$ resonance excitation. Since $N^{*}(1535)$ is the lowest energy baryonic resonance having an appreciable branching ratio for the decay into the $N \eta$ channel, its dominance in this reaction even at beam energies near the $\eta$ production threshold is to be expected. The contribution of $N^{*}(1650)$ resonance state is small and that of the $N^{*}(1710)$ resonance is even smaller at these lower beam energies. It should, however be noted that the resonance-resonance interference terms are not negligible. For the $p p \rightarrow p p \eta$ reaction, the total cross sections are smaller than those corresponding to the $N^{*}(1535)$ resonance alone. For the $p n \rightarrow p n \eta$ reactions the difference between the two is not visible in Fig. 8 .

We found that the inclusion of the amplitudes corresponding to the nucleon intermediate 
states (the nucleon bremsstrahlung) made a negligible difference in the results reported in Figs. 1-8 if the value of the coupling constant $g_{N N \eta}$ is taken below 3.0. With the largest value of $g_{N N \eta}$ used in the literature (6.14), the results were affected to the extent of only a few percent. This result is in agreement with that reported in Ref. [29]. It is obvious that due to a considerable amount of uncertainty in the value of $g_{N N n}$ (see, e.g. [48, 75, 76]), the nucleon excitation amplitudes are quite uncertain (see, e.g. [48, 75, 76]) and their inclusion makes an insignificant difference to the results reported above.

In Fig. 9, we show contributions of various meson exchanges to the $p p \rightarrow p p \eta$ reaction at near threshold beam energies. The dashed, dotted, dashed-dotted and dashed-double dotted curves represent the contributions of $\pi, \rho, \sigma$ and $\omega$ meson exchanges, respectively. Their coherent sum is shown by the solid line. We see that the one pion exchange graphs make the largest contribution to the reaction in this energy regime. However, a striking feature of this figure is that despite a larger value for the $g_{N^{*} N \rho}$ coupling used in our calculations, the contributions of $\rho$ meson exchange is still much smaller than that of the pion exchange graphs. Hence in contrast to the results reported in Refs. [67, 77] the $\rho$ meson exchange terms do not dominate the total $N N \eta$ production cross sections. To understand this difference we note that while in Refs. [67, 77] $\gamma_{5} \gamma_{\mu}$ couplings have been used for the $\rho N N^{*}$ vertex, we have taken a $\gamma_{5} \sigma_{\mu \nu}$ coupling for the same which is an extension of the $\gamma N N^{*}$ couplings (due to vector meson dominance reasons [48]). This is also compatible with the forms of the $\rho N N^{*}$ couplings used in the literature [46, 78, 79]. Since the $\rho$ meson exchange amplitudes calculated with the $\gamma_{5} \sigma_{\mu \nu}$ couplings involve delicate cancellations among leading terms, contributions of this exchange diagrams to the $\eta$ production cross sections are weakened. This is the main reason for differences between our results and those of Refs. [67, 77].

In Ref [29], although the form of the $\rho N N^{*}$ coupling is the same as ours, relatively lower $\rho$ meson exchange cross sections result from the use of a very small value for the coupling constant $g_{N^{*} N \rho}$ which is based on the lower limit of the branching ratio for the radiative decay of this resonance. Our value for this constant, on the other hand, is calculated from the branching ratio of the decay of this resonance to $N \rho$ channel as quoted in Ref. [52].

We see that the $\omega$ meson exchange process contributes insignificantly to the $N N \eta$ production but the $\sigma$ meson exchange terms are relatively more important. Larger contributions from the latter has also been seen in other subthreshold reactions analyzed within our model. It indicates that $\sigma$ meson exchange may be an efficient means of mediating the large mo- 


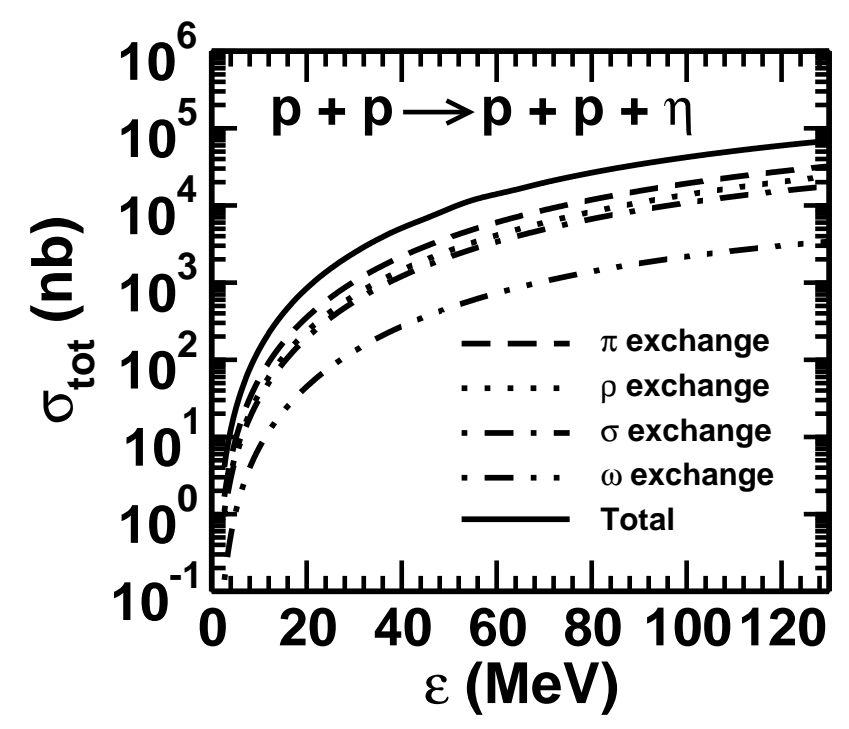

FIG. 9: Contributions of $\pi$ (dashed line), $\rho$ (dotted line), $\omega$ (dashed-double dotted line) and $\sigma$ (dashed-dotted line) meson exchange processes to the total cross section for the $p p \rightarrow p p \eta$ reaction as a function of the excess energy. Their coherent sum is shown by the solid line.

mentum mismatch involve in the meson production in $N N$ collisions [80, 81].

In fig. 10, we investigate the effects of using PS or PV couplings for the $N^{*} N \pi(\eta)$ vertices. We notice hardly any difference in the cross sections calculated by the two types of couplings. Similar results were also observed in Ref [46]. This result is not surprising since the two couplings are constructed in such a way that both are equivalent on the mass shell. Of course, they start having different energy behavior in the far off shell region where resonance contribution is anyway suppressed due to dominance of the corresponding propagator. It is only in the $N N \pi$ case that difference in the PS and PV couplings are obvious with a clear preference for the PV coupling in line with the chiral symmetry as discussed earlier.

After establishing the dynamical content of our model vis a vis the description of the total production cross sections, we now turn our attention to more exclusive data. In Fig. 11, we show a comparison of our calculations with the data for the angular distribution of $\eta$ meson in the $p p \rightarrow p p \eta$ reaction for $\epsilon$ values of $15 \mathrm{MeV}$ (upper panel) and $41 \mathrm{MeV}$ (lower panel). Since the angular distribution data are normalized to the total cross sections for both values of $\epsilon$, we have done the same in our calculations shown in this figure. We note that shapes of the angular distributions are described well by our model at both the energies. At the lower beam energy, the data as well as our calculations have essentially isotropic 


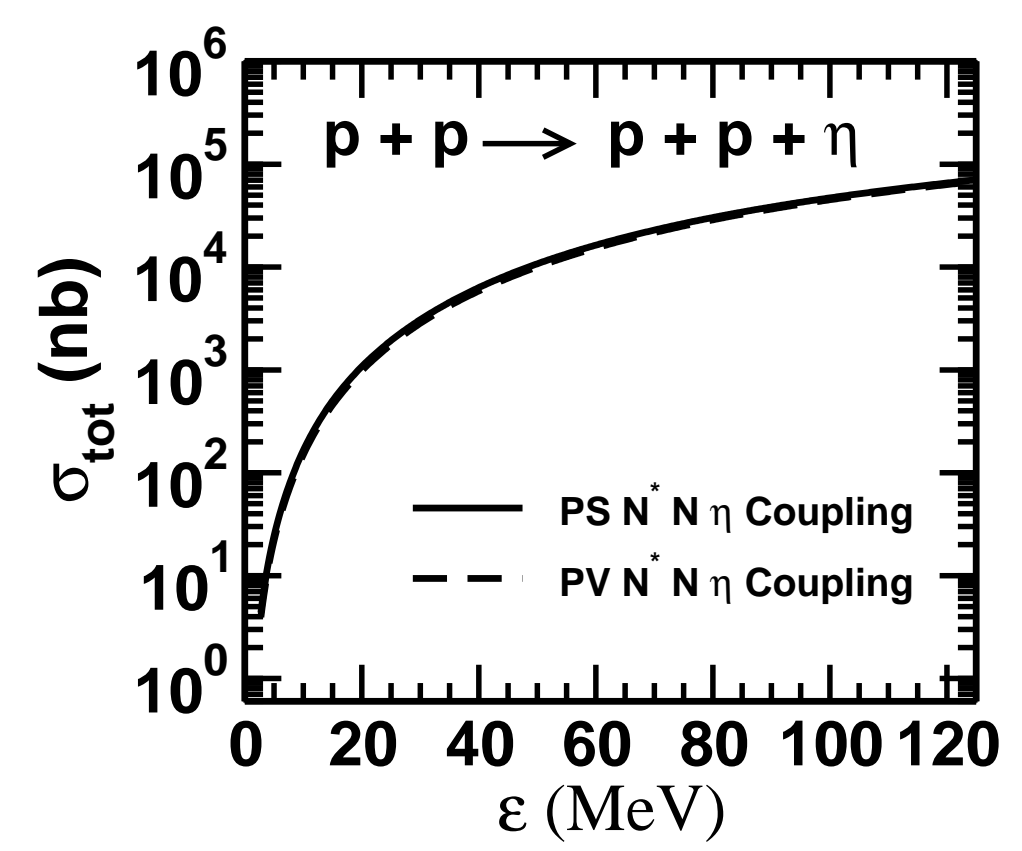

FIG. 10: The total cross section for the $p p \rightarrow p p \eta$ reaction calculated with pseudovector (solid line) and psuedoscalar (dashed line) couplings for the $N^{*} N \pi(\eta)$ vertex for the resonances considered in this paper, as a function of the excess energy.

distributions. However, for $\epsilon=41 \mathrm{MeV}$, there is a tendency in our calculations to show slight enhancements at forward and backward angles which is typical of the $\pi$ exchange dominance process in the $N^{*}(1535)$ excitation. Due to large statistical errors in the data, it is difficult to conclude if they show a trend different from our calculations. It will be useful to have better quality data with less statistical errors in order to determine if other mechanisms which may show a trend different from ours, are also important. In any case, it is quite unlikely that the $\rho$ meson exchange mechanism which might lead to a distribution different from that seen in our calculations [28] is a dominant mechanism as has already been discussed.

\section{SUMMARY AND CONCLUSIONS}

In this paper we investigated the $\eta$ meson production in proton-proton and protonneutron collisions for beam energies ranging from near threshold to about $10 \mathrm{GeV}$ within an effective Lagrangian model which has been used previously to describe successfully the pion, associated kaon and dilepton production in $N N$ collisions. The interaction between two 


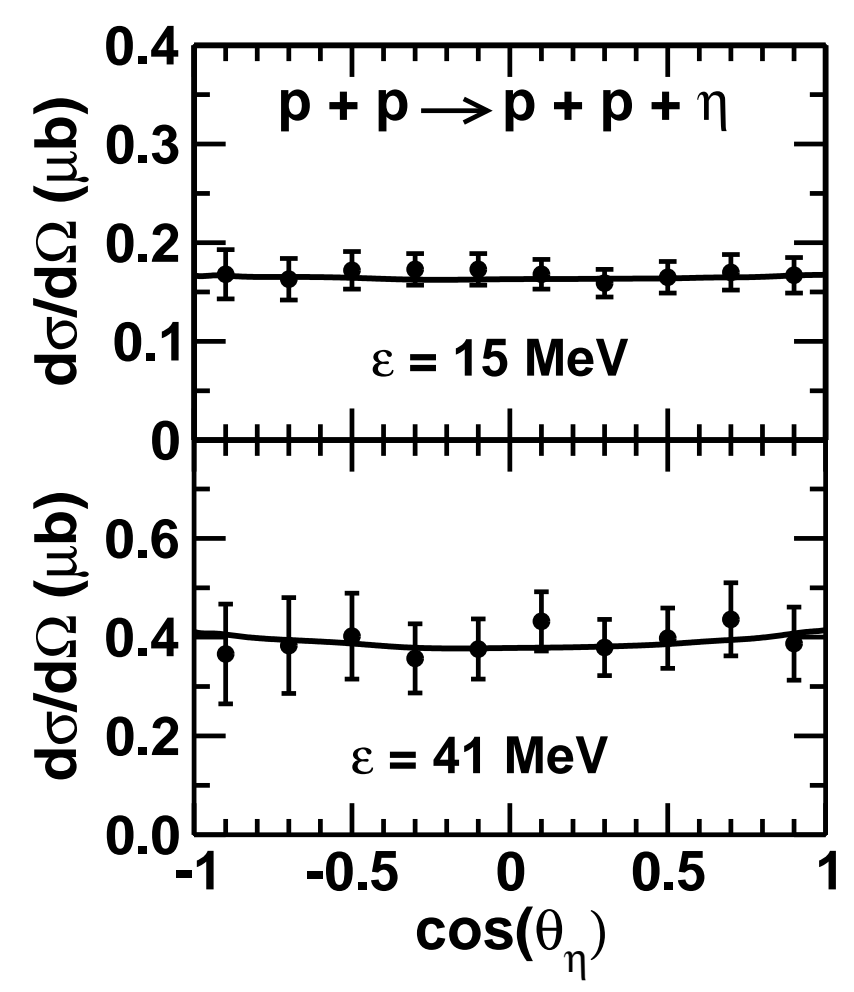

FIG. 11: Differential cross sections of the $p p \rightarrow p p \eta$ reaction as a function of $\eta$ meson angle in the c.m. frame of the total system at the excess energies of $15 \mathrm{MeV}$ (upper panel) and $41 \mathrm{MeV}$ (lower panel). The experimental data have been taken from the Ref. [8].

nucleons in the initial state is modeled by the effective Lagrangians based on the exchange of $\pi, \rho, \omega$ and $\sigma$ mesons. The parameters of the corresponding vertices were taken to be the same as those used in the previous applications of this model which restricts the freedom of varying the parameters to get fits to the data. The eta meson production proceeds via excitation, propagation and decay of $N^{*}(1535), N^{*}(1650)$ and $N^{*}(1710)$ intermediate nucleon resonance states. The coupling constants at the resonances-nucleon-meson vertices have been determined from the experimental branching ratios of the decays of the resonances into the relevant channels. Here again we have used the same coupling constants as those used in the previous applications of the model at vertices that appeared also in those calculations. The interference terms among various amplitudes are included in the total transition matrix.

To describe the data at the near threshold beam energies, the FSI effects among the outgoing particles are included by a generalized Watson-Migdal method which allows to have these effects in all the three two body sub-systems of the out-going channel. This 
method involves a parameter which has been determined from the constrained that in the limit of FSI in only $N N$ sub-system the result of the usual Watson method is reproduced.

In this paper we presented the analysis of the data for total cross sections for the $p p \rightarrow p p \eta$ and $p n \rightarrow p n \eta$ reactions and for the $\eta$ angular distributions in the former reaction. With the same set of vertex parameters, the model is able to provide a good description of the data for the $p p \rightarrow p p \eta$ reaction at higher as well as near threshold beam energies except for the excess energies below $15 \mathrm{MeV}$ where our calculations underpredict the experimental data. The experimental total cross sections of the $p n \rightarrow p n \eta$ reaction are also well described by our model. The data for the $\eta$ angular distributions in the case of $p p \rightarrow p p \eta$ reaction are also well reproduced at two beam energies. Imprecise knowledge of the $\eta N$ scattering amplitudes and non-inclusion of the three-body effects are the most likely reasons for underestimation of the $p p$ channel data by our model at very low beam energies.

In this study we have not studied the observables related to meson energy dependence e.g., final $p p$ and $\eta p$ invariant mass distributions. There are some open theoretical issues concerning the explanation of the corresponding experimental data. While in Ref. [30], the inclusion of contributions of the non-s-wave states in the $p p$ subsystem were found to be essential to explain these data, the three-body effects in the $p p \eta$ system and not the contribution of the higher partial waves were shown to be crucial for this purpose by the authors of Ref. [32]. At this stage, our theory excludes both these effects. Extension of our model to include these mechanisms is vital before we can make some meaningful contribution towards settlement of this issue.

Within our model, one pion exchange processes make the largest contributions to cross sections in the entire energy regime. Despite our using a large coupling constant for the $N^{*}(1535) N \rho$ vertex, the cross sections of the $\rho$ meson exchange process are still lower than those of the pion exchange mechanism. Therefore, $\rho$ meson exchange being the dominant mechanism of $N^{*}(1535)$ resonance excitation [28] is not supported by our calculations. The individual contributions of the $\omega$ meson exchange diagrams are very small every where. On the other hand, the $\sigma$ exchange terms make relatively larger contributions.

The excitation of the $N^{*}(1535)$ resonance dominates the $N N \eta$ production at both higher as well as near threshold beam energies. The contributions of $N^{*}(1650)$ and $N^{*}(1710)$ are small in comparison. However, the interference among various resonance contributions is not negligible. Unlike the $N N \pi$ vertex where there is a clear preference for the PV 
coupling, the present reaction does not distinguish between PS and PV couplings at the $N^{*} N \eta$ vertex involving spin-1/2 even or odd parity resonances. We point out that the mechanism of the $p p \eta$ production via preferential excitation of the $N^{*}(1535)$ intermediate baryonic resonance state in one-pion-exchange process has received support recently from an experimental study [82] of the analyzing powers of the $\bar{p}+p \rightarrow p+p+\eta$ reaction.

This work fixes the parameters of the effective Lagrangian model for most of the vertices involve in the eta meson production processes. An interesting further check of this model will be provided by the analysis of the eta photoproduction data on nucleons (see,e.g. [83, 84]). An exciting recent result is that the integrated cross section of the photoproduction of $\eta$ meson on neutrons shows an additional maximum at center of mass energies around 1.66 $\mathrm{GeV}$. This has recently been explained in terms of the excitation of the $N^{*}(1650)$ and $N^{*}(1650)$ resonance states [85]. Furthermore, the vertex parameters derived by us will also be useful in applications of effective Lagrangian method in describing the production of eta-mesic nuclei in proton and photon induced reactions (see, e.g. [86, 87]).

\section{ACKNOWLEDGMENTS}

The author is thankful to Andrzej Deloff for several useful correspondences regarding the off shell $p p$ FSI method presented in Ref. [61] and for his help in implementing the calculations based on this method. Useful discussions with Horst Lenske, Jerry Miller, Ulrich Mosel, Pawel Moskal and Madeleine Soyeur is gratefully acknowledged.

\section{APPENDIX A: FINAL STATE INTERACTION AMPLITUDES FOR THREE- PARTICLE STATES}

We give here some clarifications and steps leading to the derivation Eq. (23).

The total Hamiltonian of the three-particle system is written as $H=H_{0}+U$ where $H_{0}$ is the kinetic energy operator of the system and the interaction $U$ is taken as $U=$ $U_{23}+U_{31}+U_{12} \equiv U_{1}+U_{2}+U_{3}$ assuming that the three-particle states interact by means of the additive pair interactions represented by $U_{k}$. The Green's functions corresponding to $H$ and $H_{0}$ are, respectively

$$
G^{( \pm)}(E)=\lim _{\epsilon \rightarrow 0} \frac{1}{E-H_{0}-U \pm i \epsilon}
$$




$$
G_{0}^{( \pm)}(E)=\lim _{\epsilon \rightarrow 0} \frac{1}{E-H_{0} \pm i \epsilon}
$$

We shall also need the Hamiltonian describing the two particles interacting while the third one is free, namely, $H_{k}=H_{0}+U_{k}$ and the corresponding Green's functions

$$
G_{k}^{( \pm)}(E)=\lim _{\epsilon \rightarrow 0} \frac{1}{E-H_{0}-U_{k} \pm i \epsilon}
$$

The full three-body transition operator $T$ satisfies the Lipmann-Schwinger equations

$$
T(E)=U+U G_{0} T(E)
$$

which can also be written as

$$
T(E)=\sum_{k} U_{k}+\sum_{k} U_{k} G_{0} T(E)
$$

Eq.(A5) leads to the iteration

$$
\begin{aligned}
T(E)= & \sum_{k}\left[U_{k}+U_{k} G_{0} U_{k}+U_{k} G_{0} U_{k} G_{0} U_{k}+\ldots\right] \\
& +\sum_{k \neq j}\left[U_{k}+U_{k} G_{0} U_{k}+\ldots\right] G_{0}\left[U_{j}+U_{j} G_{0} U_{j}+\ldots\right]+\ldots
\end{aligned}
$$

In the study of the final state interaction problems, one usually has in addition to potential $\mathrm{U}$ [which is responsible for the transition from the initial state to the free final states characterized by the transition matrix $T_{0}$ in Eq. (22)], an additional interaction $\mathrm{V}$ that describes a type of internal interaction among the constituents of the final state. The total Hamiltonian is then written as $H_{0}+U+V$ and one can make use of the standard "twopotential formalism" of Goldberger and Watson (see, e.g., [42]) to write the total scattering matrix element $\left(T_{f i}\right)$ as as a sum of two terms - one of them involve the matrix elements of the interaction $U$ between the exact initial state wave function and the final scattering state wave function corresponding to interaction $V$. From the general theory, we can write

$$
T_{f i}=<\chi_{f}|U+V| \psi_{i}^{(+)}>
$$

where $\psi_{i}^{(+)}$completely describes the initial state with the outgoing wave boundary condition, and $\chi_{f}$ is the final plane wave state. One can eliminate $\chi_{f}$ by introducing wave functions $\phi_{f}^{(-)}$which are the eigenfunctions of the Hamiltonian $H_{0}+V$ as

$$
\phi_{f}^{(-)}=\chi_{f}+G_{0}^{(-)} V \phi_{f}^{(-)}
$$


Substituting (A8) into (A7) one gets after some manipulation,

$$
T_{f i}=<\phi_{f}^{(-)}|U| \psi_{i}^{(+)}>+<\phi_{f}^{(-)}|V| \chi_{i}>
$$

where $\chi_{i}$ is the initial plane wave state. The final state interactions of interest are contained in $\phi_{f}^{(-)}$.

In applications of relevance to us, the second term of Eq. (A9) would vanish because we assume $V$ can not create real mesons. Eq. (23) can be obtained by using an iteration similar to that given by Eq. (A6) in the remaining (first) term of Eq. (A9). The amplitude $T_{0}$ is defined in the same way as the first term of Eq. (A9) with a plane wave final state. The amplitude $T_{k}$ in the second term of Eq. (23) represents the matrix elements of the interaction $V_{k}$ between the plane wave and the scattering states of particle $i j$. We define $V_{k}$ for the partition $k$ in the same way as $U_{k}$.

[1] K. G. Wilson, Phys. Rev. D10, 2445 (1974).

[2] D. B. Leinweber, W. Melnitchouk,,D. G. Richards, A. G. Williams, and L. M. Zanotti, arXiv:nucl-th/0406032; J. M. Zanotti, B. Lasscock, D.B. Leinweber and A. G. Williams, Phys. Rev. D71, 034510 (2005); R.D. Young, D. B. Leinweber, and A. W. Thomas, Phys. Rev. D71, 014001 (2005).

[3] P. Mosal, M. Wolke, A. Khoukaz, and W. Oelert, Progr. Part. Nucl. Phys. 49, 1 (2002)

[4] H. O. Meyer et al., Phys. Rev. C 63, 064002 (2001); and references therein.

[5] J. Balewski et al., Phys. Lett. B420, 211 (1998).

[6] S. Sewerin et al., Phys. Rev. Lett. 83, 682 (1999).

[7] P. Kowina et al., Eur. Phys. J, A 22, 293 (2004).

[8] M. Abdel-Bary et al., Phys. Lett. B595, 127 (2004).

[9] A. Abd El-Samad et al., Phys. Lett. B632, 27 (2006)

[10] H. Calen et al., Phys. Rev. C. 58, 2667 (1998).

[11] H. Calen et al., Phys. Lett. B 458, 190 (1999).

[12] J. Smyrski et al., Phys. Lett. B 474, 182 (2000); F. Hibou et al., ibid. 438, 41 (1998); H. Calen et al., ibid. 366, 39 (1996); E. Chiavassa et al., ibid. 322, 270 (1993); A.M. Bergdolt et al., Phys. Rev. D 48, R2969 (1993). 
[13] M. Abdel-Bary et al., Eur. Phys. J. A 16, 127 (2003)

[14] F. Balestra et al., Phys. Rev. C 69, 064003 (2004).

[15] P. Moskal et al., Phys. Rev. C 69, 025203 (2004).

[16] C. Dover and P. Fishbane, Phys. Rev. Lett. 64, 3115 (1990).

[17] See, e.g., Z. Papandreou, Eleventh International conference on Hadron spectroscopy, Rio de Janeiro (Brazil), August 21-26, 2005, AIP conference Proceedings, Vol. 814, page 453.

[18] N. Mathur, Y. Chen, S. J. Dong, T. Draper, I. Horvath, F. X. Lee, K. F. Liu, and J. B. Zhang, Phys. Lett. B 605, 137 (2005); C. Hanhart, Acta Phys. Slov. 56, 193 (2005) (arXiv:nucl-th/0511045).

[19] H.C. Chiang, E. Oset, and L.C. Liu, Phys. Rev. C 44, 738 (1991).

[20] A. Fix and H. Arenhövel, Phys. Rev. C 66, 024002 (2002).

[21] Q. Haider and L.C. Liu, Phys. Rev. C 66, 045208 (2002).

[22] M. Pfeiffer et. al., Phys. Rev. Lett. 92, 252001 (2004).

[23] A. Sibirtsev, J. Haidenbauer, J.A. Niskanen, and Ulf-G. Meissner, Phys. Rev. C 70, 047001 (2004).

[24] International workshop on $\eta$-nucleus Physics, May 8-12, 2006, Jülich, Germany, summary is available at arXiv:nucl-th/0610011.

[25] W. Cassing, V. Metag, U. Mosel and K. Niita, Phys. Rep. 188, 363 (1988).

[26] T. Vetter, A. Engel, T. Biro, and U. Mosel, Phys. Lett B 263, 153 (1991).

[27] A. Moalem, E. Gedalin, L. Razdolskaja, and Z. Shorer, Nucl. Phys. A600, 445 (1996); E. Gedalin, A. Moalem, L. Razdolskaya, Nucl. Phys. A634, 368 (1998); ibid., Nuc. Phys. A 650, 471 (1999).

[28] G. Fäldt and C. Wilkin, Phys. Scr. 64, 427 (2001).

[29] K. Nakayama, J. Speth, and T. -S. H. Lee, Phys. Rev. C 65, 045210 (2002).

[30] K. Nakayama, J. Haidenbauer, C. Hanhart, and J. Speth, Phys. Rev. C 68, 045201 (2003).

[31] V. Baru, H. Haidenbauer, C. Hanhart, Y. Kalashnikova and A. Kudryavtsev, Phys. Lett. B 586, 53 (2004).

[32] A. Fix, and H. Arenhövel, Phys. Rev. C 69, 014001 (2004),

[33] A. Engel, R. Shyam, U. Mosel, and A. K. Dutt-Majumder, Nucl. Phys. 603, 387 (1996).

[34] R. Shyam and U. Mosel, Phys, Lett. B425, 1 (1998).

[35] R. Shyam, Phys. Rev. C 60, 055213 (1999); R. Shyam, G. Penner, and U. Mosel, Phys. Rev. 
C 63, 022202(R) (2001); R. Shyam, Phys. Rev. C 73, 035211 (2006).

[36] R. Shyam, and U. Mosel, Phys. Rev. C 67, 065202 (2003).

[37] M. Schäfer, H. C. Dönges, A. Engel, and U. Mosel, Nucl. Phys. A575, 429 (1994).

[38] S. Weinberg, Phys. Rev. 166, 1568 (1968).

[39] V. Bernard, N. Kaiser, and Ulf-G. Meissner, Int. J. Mod. Phys. E 4, 193 (1995).

[40] C. Hanhart, Phys. Rep. 397, 155 (2004).

[41] R. Machleidt, K. Hollinde and Ch. Elster, Phys. Rep. 149, 1 (1987).

[42] M.L. Goldberger and K.M. Watson, Collision Theory, Wiley, New York, 1969, pp 549.

[43] J. Gillespie, Final-State Interaction, Ed. K. M. Watson, Holden-Day Adv. Phys. Monographs, Holden-Day, San Francisco, 1964, and references therein.

[44] A. Deloff, arXiv:nucl-th/0406069.

[45] T. Ericson and W. Weise, Pions and Nuclei (Clarendon, Oxford, 1988).

[46] G. Penner and U. Mosel, Phys. Rev. C 66, 055211 (2002); 66, 055212 (2002); G. Penner, Ph.D. thesis (in English), Universität Giessen, 2002, available at the URL http://theorie.physik.uni-giessen.de

[47] J. D. Bjorken and S. D. Drell, Relativistic Quantum Mechanics (McGraw-Hill, New York, 1964).

[48] M. Benmerrouche, N.C. Mukhopadhyay, and J.F. Zhang, Phys. Rev.D 51, 3237 (1995).i

[49] W. Grein and P. Kroll, Nucl. Phys. A338, 332 (1980).

[50] C. Bennhold, Nucl. Phys. A585, 313c (1995).

[51] M. Kirchbach and L. Tiator, Nucl. Phys. A605, 385 (1996).

[52] W.M. Yao et. al. (particle data group), J. Phys. G: Nucl \& Part. Phys. 33, 1 (2006).

[53] T. Feuster and U. Mosel, Nucl. Phys. A 612, 375 (1997).

[54] T. Feuster and U. Mosel, Phys. Rev.C 58, 457 (1998).

[55] R. Shyam, H. Lenske, and U. Mosel, Nucl. Phys. A 764, 313 (2006).

[56] J.J. Sakurai, Currents and Mesons, Univ. of Chicago Press, Chicago, 1969; Ann. Phys. 11, 1 (1960).

[57] J. Dubach, W.M. Kloet, and R.R. Silbar, Phys. Rev. C 33, 373 (1986).

[58] V. Bernard, N. Kaiser, and Ulf-G. Meissner, Eur. Phys. J. A 4, 259 (1999).

[59] A. Moalem, E. Gedalin, L. Razdolskaja, and Z. Shorer, Nucl. Phys. A 600, 455 (1996).

[60] B.L. Druzhinin, A.E. Kudryavtsev, and V.E. Tarasev, Z. Phys. A 359, 205 (1997). 
[61] A. Deloff, Phys. Rev. C 69, 035206 (2004).

[62] A. Sibirtsev, J. Haidenbauer, H.-W. Hammer, U.-G. Meissner, Eur. Phys. J. A29, 363 (2006).

[63] A.I. Titov, B. Kämpfer, and B.L. Reznik, Eur. Phys. J. A 7, 543 (2000).

[64] M. Soyeur and M.F.M. Lutz, Int. J. Mod. Phys. A22, 333 (2007) (arXiv:nucl-th/0607019).

[65] A. M. Green, and S. Wycech, Phys. Rev. C 71, 014001 (2005).

[66] M.F.M. Lutz, Gy. Wolf, B. Friman, Nucl. Phys. A 706, 431 (2002).

[67] E. Gedalin, A. Moalem, L. Razdolskaya, Nucl. Phys. A634, 368 (1998); ibid., Nuc. Phys. A 650, 471 (1999).

[68] H. Garcilazo and M. T. Pena, Phys. Rev. C 66, 034606 (2002).

[69] A. M. Gasparyan, J. Haidenbauer, C. Hanhart, and J. Speth, Phys. Rev. C 68, 045207 (2003).

[70] H. P. Noyes, Annu. Rev. Nucl. Sci, 22, 465 (1972).

[71] C. Hanhart and K. Nakayama, Phys. Lett. B454, 176 (1999).

[72] V. Baru, A. M. Gasparyan, J, Haidenbauer, A. E. Kudryavtsev, and J. Speth, Phys. At. Nucl. 64, 579 (2001) [Yad. Fiz. 64, 633 (2001)].

[73] A. Gasparyan, J. Haidenbauer, and C. Hanhart, Phys. Rev. C 72, 034006 (2005).

[74] Landolt-Börnstein: Numerical Data and Functional Relationships in Science and Technology, New Series, edited by H. Schopper, I/12 (Springer, Berline, 1988)

[75] B. Lopez Alvaredo and E. Oset, Phys. Lett. B 324, 125 (1994).

[76] W.-T. Chiang, S. N. Yang, L. Tiator, and D. Drechsel, Nucl. Phys. A700, 429 (2002).

[77] A. B. Santra and B. K. Jain, Nucl. Phys. A634, 309 (1998).

[78] M. Post, S. Leupold, and U. Mosel, Nucl. Phys. A 689, 753 (2001).

[79] D. O. Riska and G. E. Brown, Nucl. Phys. A679, 577 (2001).

[80] T. S. H. Lee and D. O. Riska, Phys. Rev. Lett. 70, 2237 (1993).

[81] C. J. Horowitz, H. O. Meyer, and D. K. Griegel, Phys. Rev. C 49, 1337 (1994).

[82] R. Czyzykiewicz et. al., arXiv:hep-ex/0611015, Phys. Rev. Lett. 98, 122003 (2007).

[83] V. Kuznetsov et al., in NSTAR 2005, Proceedings of the workshop on Physics with Excited Nucleons, edited by S. Capstick, V. Crede, and P.M. Eugenio, (World Scientific, Singapore, 2006) (arXiv:hep-ex/0601002).

[84] B. Krusche et al., Talk given at International workshop on $\eta$ Nucleus Physics, May 8-12, 2006, Jülich, Germany, arXiv:nucl-th/0610011.

[85] V. Shklyar, H. Lenske, and U. Mosel, arXiv:nucl-th/0611036. 
[86] M.Kh. Anikina et al., arXiv:nucl-ex/0412036.

[87] V.A. Baskov, J.P. Bocquet, V. Kouznetsov, A. Lleres, A.I. L'vov, L.N. Pavlyuchenko, V.V. Polyanski, D. Rebrevend, G.A. Sokol, arXiv:nucl-ex/0306011. 